\title{
Chapter 17 \\ The Use of Retroviral Pseudotypes for the Measurement of Antibody Responses to SARS Coronavirus
}

\author{
Nigel James Temperton
}

\begin{abstract}
Neutralization assays allow for sensitive detection of functional antibody responses directed against the surface protein envelopes of many viruses. For highcontainment viruses like SARS coronavirus (CoV), however, these assays are not widely applicable due to the requirement for high biosafety laboratory facilities and specially trained personnel. In order to effectively address this containment issue, retroviral pseudotypes have been used as surrogates of the live virus for neutralization assays. The pseudotype-based neutralization assay system is highly flexible, allowing for a choice of reporter systems, and is readily adaptable to newly emerging virus strains.
\end{abstract}

\subsection{Introduction}

The coronavirus that causes severe acute respiratory syndrome (SARS-CoV) is a relatively new human pathogen for which a vaccine will be urgently required should this virus reemerge and new outbreaks occur.

The SARS-CoV genome encodes four structural proteins, the spike (S), membrane (M), envelope (E), and nucleocapsid (N) proteins. The $S$ protein is the major surface antigen of the virus, and the antibody response is primarily directed against this protein. Preclinical studies of SARS-CoV vaccines provide evidence that generating a strong neutralizing antibody response to SARS-CoV S may protect against SARS infection. Many methods of detecting and measuring antibody responses against SARS-CoV have been developed and include IFA, ELISA, ICT, Western blot and virus neutralization (Bermingham et al. 2004; Wu et al. 2004).

\footnotetext{
N.J. Temperton

MRC/UCL Centre for Medical Molecular Virology, University College London, Windeyer Building, 46 Cleveland Street, London W1T 4JF, United Kingdom

e-mail: nigel.temperton@ucl.ac.uk
} 
Neutralization assays allow for sensitive detection of functional antibody responses directed against the surface protein envelopes of many viruses. For high-containment viruses like SARS-CoV and influenza H5N1, however, these assays are not widely applicable due to the requirement for high biosafety laboratory facilities and specially trained personnel. In order to effectively address this containment issue, retroviral pseudotypes have been used as alternatives to the live virus. These pseudotypes carry the retroviral RNA genome coding for marker genes (GFP, luciferase and beta-galactosidase ( $\beta$-gal) are the most frequently used). The RNA genome is packaged by retroviral core proteins, but these viral cores bear foreign, often nonretroviral envelope proteins on their surface. Pseudotype infection, i.e. transfer of their genome (containing the transfer/marker gene) to target cells, depends on the function of the foreign envelope proteins and results in integration and subsequent expression of the marker gene. The function of the foreign envelope can therefore be detected/quantified by measuring marker gene expression. Using retroviral particles pseudotyped with the envelopes of highcontainment viruses as "surrogate viruses" for use in neutralization assays is highly advantageous. Using a pseudotype approach, only the envelope protein(s) of the high-containment virus is required, with no possibility of recombination or virus escape. These pseudotypes undergo abortive replication and are unable to give rise to replication-competent progeny (Temperton and Wright 2009).

Safety considerations aside, for virus neutralization assays, pseudotypes are excellent serological reagents as they encode reporter genes and bear the heterologous viral envelope proteins of interest. The transfer of these reporter genes to target cells depends on the function of the viral envelope protein; therefore the titre of neutralizing antibodies against the envelope can be measured by a reduction in reporter gene transfer. These assays are also readily adaptable to a high-throughput format for use in vaccine evaluation, antiviral screening/resistance assays, monoclonal antibody screening and large sero-surveillance studies.

\subsection{Construction of Retroviral Pseudotypes Bearing SARS-CoV (S) Glycoproteins}

SARS pseudotypes are routinely constructed using a three-plasmid transfection approach (Fig. 17.1). Construction of retroviral pseudotypes is achieved through the concurrent introduction of a SARS spike $(S)$ envelope gene, core retroviral genes and a marker/reporter gene into producer cells, normally HEK 293T cells as they transfect efficiently, although other cell lines may be used;

Plasmid 1: The SARS spike (S) envelope gene construct. SARS spike (S) envelope sequences (either native or codon-optimized) can be readily cloned into appropriate expression plasmids, which will permit their incorporation into the mature pseudotype. Expression plasmids that have been successfully used for this purpose include pCAGGS (Simmons et al. 2004; Temperton et al. 2005), 


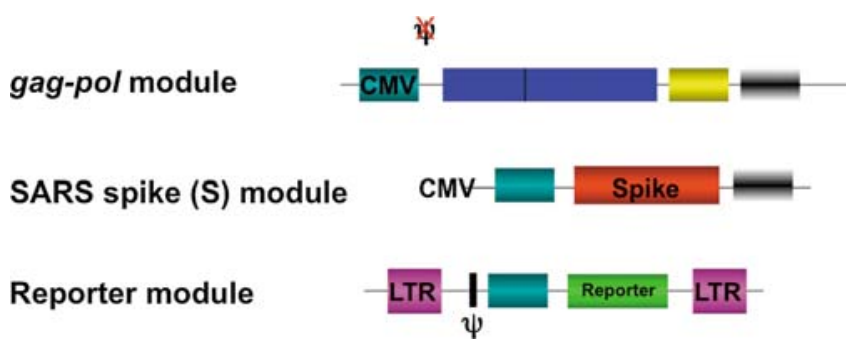

Fig. 17.1 Flexible 3 plasmid transfection system for the production of SARS-CoV (S) pseudotype particles. gag-pol module: The retroviral structural ( $\mathrm{gag}$ ) and enzymatic ( $\mathrm{pol}$ ) proteins are expressed from a single plasmid lacking the packaging signal (removed to prevent incorporation into mature particles). SARS spike (S) module: Spike envelope glycoprotein expression is driven by a promoter specific to the envelope. Transfer/reporter module: GFP/luciferase or $\beta$-gal transfer genes are cloned between flanking LTR regions that play a role in integration and transcription of the pseudotype's RNA genome. A packaging signal $(\boldsymbol{\psi})$ and promoter are incorporated upstream of the transfer gene to ensure it is packaged into the pseudotype particle and regulates expression once the gene is integrated

pcDNA3.1+ (Nie et al. 2004a; Zhang et al. 2006), pHCMV (Giroglou et al. 2004; Han et al. 2004) and pcDM8 (Moore et al. 2004).

Plasmid 2: The retroviral gag-pol construct. These are the two genes that encode the structural proteins (e.g., matrix, nucleocapsid and p7 expressed from gag) that comprise the core, and the enzymatic proteins (reverse transcriptase, protease and integrase expressed from $\mathrm{pol}$ ) responsible for processing the structural proteins and ensuring integration of a dsDNA copy of the marker/reporter gene. Additionally in a lentiviral gag-pol construct, the rev gene is included for efficient processing.

Plasmid 3: The marker/reporter gene construct. This is the gene that is stably integrated into the target/assay cell DNA. Where it is expressed via a single plasmid, a packaging signal is engineered upstream of the gene to ensure efficient incorporation of full-length RNA copies of that gene into the pseudotype capsid. Once integrated, the gene is expressed via various cis-acting transcriptional elements. It should be noted that the marker/reporter gene construct is not required for pseudotype production and mature particles will be produced by a two-plasmid transfection of a gag-pol construct and a SARS spike (S) envelope construct. Also, pseudotypes lacking the SARS spike (S) envelope will bud but will be noninfectious.

As an alternative to using the three-plasmid transfection system for producing SARS pseudotypes, all of the necessary genes can be transfected via either one or two plasmids. Using three plasmids as detailed here, however, offers the greatest ease and flexibility (switch of plasmids, minimal cloning required), and safety (less chance of recombination) as the envelope, core and reporter genes are expressed from different plasmids. The flexibility of the system enables tailoring construction of the SARS pseudotypes depending on experimental requirements. After transfection the pseudotype virus is harvested and stored at $-80^{\circ} \mathrm{C}$ until required for neutralization assays (Fig. 17.2). 


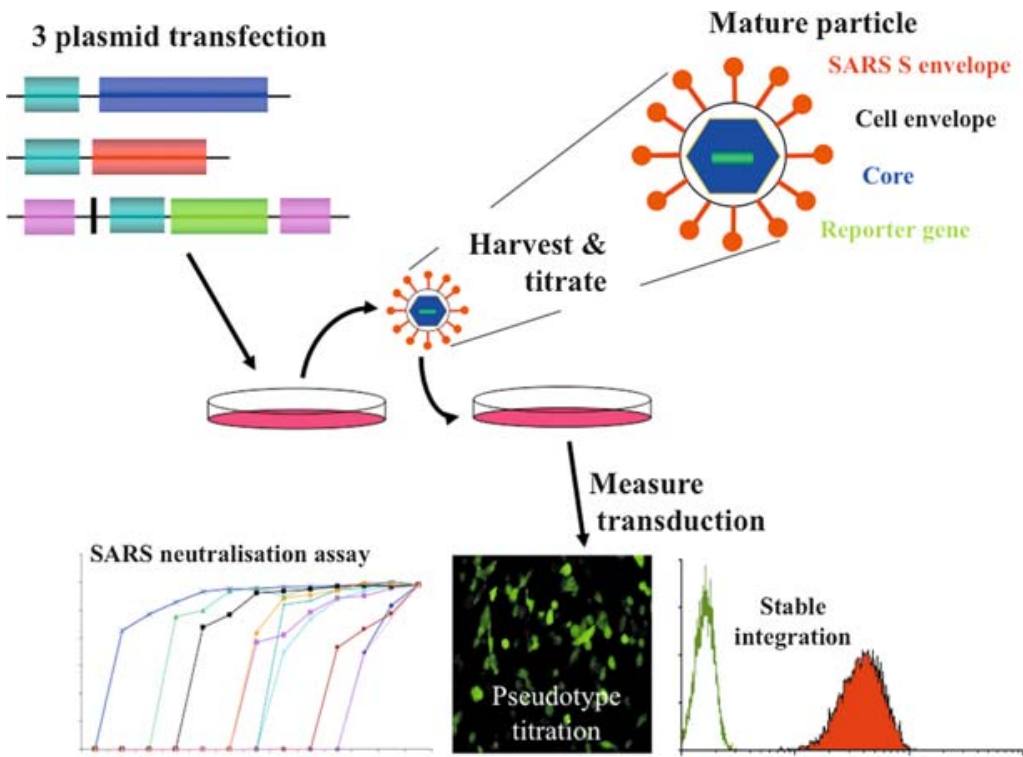

Fig. 17.2 SARS-CoV S pseudotype production, titration and neutralization. Three plasmids (gagpol module, SARS spike module and transfer/reporter module) are transfected into producer cells and the resulting mature pseudotype particles are harvested 48 and $72 \mathrm{~h}$ post-transfection. These particles comprise a core encasing the RNA genome, surrounded by an outer matrix protein complex, with the producer cell plasma membrane displaying the SARS-CoV S envelope proteins, forming the pseudotype virus membrane. These mature pseudotype particles can subsequently be loaded onto target cells and the level of transduction/infection measured using approprate methodologies (FACS, fluorescence microscopy, luminometry)

\subsection{Neutralization Assay}

A neutralization assay using SARS pseudotypes is performed routinely as follows: patient serum samples are heat-inactivated at $56^{\circ} \mathrm{C}$ for $30 \mathrm{~min}$, serially diluted in appropriate culture medium, and mixed with SARS pseudotype virus at a 1:1 volume/volume ratio in a 96-well flat-bottomed tissue culture plate (clear plastic for GFP and $\beta$-gal, white plastic for luciferase). After incubation at $37^{\circ} \mathrm{C}$ for $1 \mathrm{~h}$, approximately $1 \times 10^{4}$ target cells are added to each well. GFP, $\beta$-gal or luciferase positive cells are counted $24-48 \mathrm{~h}$ later, and neutralizing antibody titers calculated (Temperton et al. 2005). Similar pseudotype-based entry assays for influenza H5N1 have been evaluated in 384- and 1,536-well tissue culture plates for highthroughput screening applications (Wang et al. 2008).

\subsection{Expanding the Repertoire of SARS-CoV (S) Pseudotypes}

In order to achieve maximum sensitivity in serological assays for vaccine evaluation, and for seroprevalence studies, the selection of virus isolated from the same SARS outbreak, or the use of an antigenically equivalent strain, is required for 
optimal antigenic match. Pseudotype viruses bearing SARS-CoV S envelopes from many different strains have been constructed and used as surrogate viruses in cell entry and neutralization assays. Hofmann et al. have constructed HIV pseudotypes using the Frankfurt strain S protein (Hofmann et al. 2004). Nie et al. have constructed HIV pseudotypes using synthetic codon-optimized S proteins from the BJ01, Frankfurt, TOR2 and TW1 strains of SARS-CoV in order to screen sera from patients with SARS (Nie et al. 2004a, 2004b). These four pseudotypes were normalized using a p24 ELISA assay. Assays were performed using human hepatoma Huh7 cells that endogenously express high levels of ACE2 receptor. Han et al. have constructed MLV pseudotypes using the Urbani strain S protein for the development of a safe neutralization assay for SARS-CoV (Han et al. 2004). Temperton et al. have constructed MLV and HIV pseudotypes using the Urbani strain $\mathrm{S}$ protein in order to profile neutralizing antibody responses to the S glycoprotein in sequential serum samples collected from recovered patients (Temperton et al. 2005). Assays were performed on quail QT6 cells stably over-expressing the ACE2 receptor. The FFM-1 strain of SARS was used by Giroglou et al. to construct MLV pseudotypes (Giroglou et al. 2004). Zhang et al. have constructed HIV pseudotypes using the HK-39 strain S protein to study neutralizing antibody responses in SARS-CoV infected patients. For these assays, a HEK293 cell line transduced with the SARS-CoV receptor ACE2 (293/ACE2) was used. Zhu et al. have used HIV pseudotypes bearing the S proteins from the TOR2, Urbani, GD03, SZ3 and SZ16 strains of SARS-CoV to study the in vitro neutralizing activity of human monoclonal antibodies (Zhu et al. 2007). Thus, to date at least ten different SARS-CoV spike glycoproteins have been incorporated successfully into retroviral pseudotypes and employed for a multitude of cell tropism and antibody assays.

Spike (S) proteins from other coronaviruses have also been efficiently incorporated into pseudotypes. Retroviral pseudotypes have been used to study receptor-mediated entry of the feline coronavirus, FCoV (Dye et al. 2007) and human coronaviruses NL63 and 229E (Hofmann et al. 2005). This powerful system is likely therefore to have pan-coronavirus applicability for virological and immunological studies.

If SARS-CoV were to reemerge and result in new human outbreaks it would be straightforward to update these neutralization assays to measure responses against newly emerging antigenic variants. Upon availability of viral RNA/cDNA or sequence information of the newly emergent SARS virus (from public nucleotide sequence databases such as the National Center for Biotechnology Information database held at http://www.ncbi.nlm.nih.gov/), the spike gene can be PCR-amplified or synthesized and retroviral pseudotypes prepared for use in downstream neutralization assays. With the cost of whole gene synthesis decreasing year on year, this is a viable proposition for the development of such serological assays, as access to the wild-type virus is often severely limited to a small group of laboratories (WHO, CDC, HPA etc.). These newly developed assays can be used to address the cross-clade neutralizing potential of candidate human vaccines and immuno-therapeutics (monoclonal antibodies etc.). They can also been used for the evaluation of anti-S virus entry inhibitors and could readily be adapted for serosurveillance studies in new outbreak locations. 


\subsection{Pseudotype Titration and Target Cell Lines}

When measuring neutralizing antibody responses against antigenically diverse SARS-CoV strains, it is necessary to accurately titre the pseudotype viruses so that virus input doses may be normalized. GFP-based pseudotypes are readily titred using flow cytometry (FACS), luciferase pseudotypes by luminometry and $\beta$-gal pseudotypes by colour reaction. The titres obtained by these methods are highly dependent on the cell type chosen for the assay, as ACE2 receptor density may differ widely between cell types. Many cell lines have been shown to be susceptible to SARS-CoV (Hattermann et al. 2005; Kaye 2006). These cell lines have been previously employed, or are predicted to be suitable target cells for use in pseudotype assays. They are BGM (buffalo green monkey kidney epithelium), COS (monkey kidney fibroblast), CV-1 (African green monkey kidney fibroblast), FRhK (rhesus monkey fetal kidney), LLC-Mk2 (rhesus monkey kidney epithelium), MA-104 (African green monkey kidney epithelium), MEK (monkey embryonic kidney), Vero (African green monkey kidney epithelium), Vero E6 (clone of Vero), HEK293 (human fetal kidney), HepG2 (human liver hepatocellular carcinoma), Huh-7 (human liver hepatocellular carconoma), Hep2 (human liver), RK-13 (rabbit kidney epithelium), POEK (porcine fetal kidney) and PS (porcine kidney).

Other methods for the titration and standardization of these retroviral pseudotypes are RT-PCR to determine virus particle number, ELISA to determine Gag levels (HIV or MLV Gag) in the retrovirus cores, and the measurement of surface envelope (SARS-CoV spike) content. Surface content may be measured by ELISA, as was recently shown for influenza H5N1 pseudotypes (Su et al. 2008).

\subsection{Reporter Systems}

In order for these pseudotype-based assays to have wide applicability within different laboratories, the availability of different reporter systems is highly desirable (Fig. 17.3). The HIV GFP reporter module pCSGW described recently for use in viral neutralization assays (Temperton et al. 2005, 2007; Wright et al. 2008) has been modified by PCR subcloning to express alternative reporters. These are firefly luciferase (Temperton et al. 2008; Wright et al. 2008), renilla luciferase (Capecchi et al. 2008) and $\beta$-gal. Of the three assay types, those based on luciferase reporter are the simplest to use in terms of operator time and data evaluation. However, due to the high cost of the necessary reagents (luciferase assay kits) and necessity for specialized equipment (luminometer), these assays may have limited applicability for laboratories in resource-poor regions. GFP and other fluorescence protein-based assays (RFP, YFP) do not require any additional assay reagents but do necessitate the availability of specialized equipment (fluorescence microscope with interchangeable colour filters, or a 96-well plate flow cytometry/FACS facility). The $\beta$-gal-based assays are the most cost-effective, as the required assay reagents are readily available at low cost, and specialized equipment is unnecessary. 
Fig. 17.3 Reporter gene readout for SARS-CoV (S) pseudotype neutralization assays. The detection of pseudotype transduction for the quantification of neutralizing antibody responses against the SARS$\mathrm{CoV}$ spike glycoprotein depends on the reporter/ transfer gene incorporated into the mature particle. Options available include GFP, luciferase and $\beta$-gal. ONPG: $\sigma$-nitrophenyl- $\beta$-Dgalactopyranoside; CPRG: chlorophenol red- $\beta$-Dgalactopyranoside

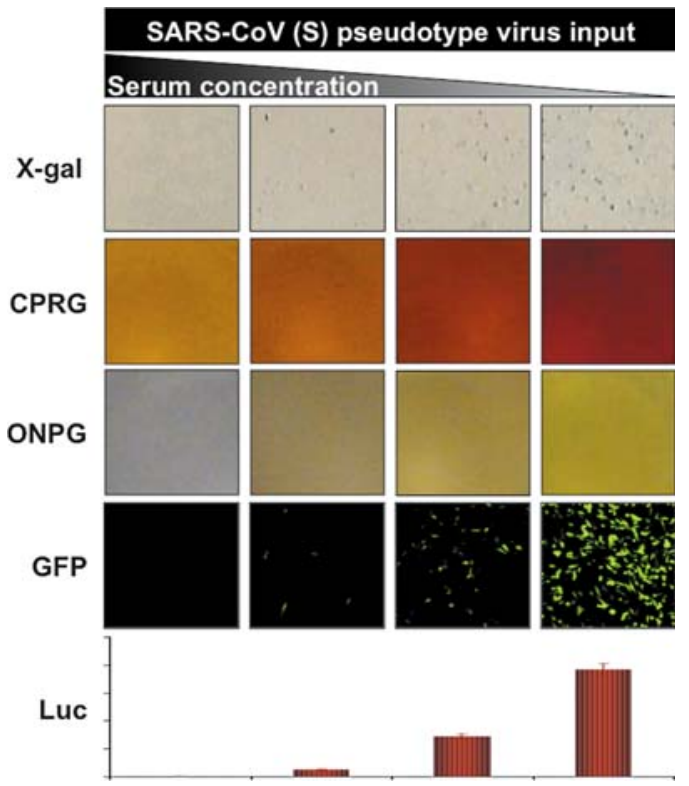

The expression of $\beta$-gal can be quantified by counting infected cells using a microscope following staining with $\mathrm{X}$-gal (5-bromo-4-chloro-3-indolyl- $\beta$-D-galactopyranoside). However, there are two colorimetric substrates, ONPG (o-nitrophenyl- $\beta$-D-galactopyranoside) and CPRG (chlorophenol red- $\beta$-D-galactopyranoside) that allow the assays to be evaluated using a microplate reader. Alternatively an automated procedure using an ELISPOT reader to count X-gal stained blue cells may be used (Han et al. 2004). Finally, the availability of multiple reporters for use in these assays also lends itself to the development of multiplex assays which would facilitate the measurement of neutralizing antibody responses against surface glycoprotein envelopes derived from two (or more) different virus families (e.g., SARS-CoV and H5N1 for sero-surveillance in wet markets).

\subsection{Correlation with Other Immunological Assays}

Once suitable SARS-CoV pseudotype neutralization assays have been established, studies can be readily performed comparing these new surrogate assays with conventional live virus neutralization assays and other parameters of immune responses to SARS-CoV infection. Nie et al. have compared titres of neutralizing antibodies in 12 serum samples analyzed with pseudovirus and live SARS-CoV neutralization assays and found a strong correlation between the two (Nie et al. 2004a). Temperton et al. showed correlation data for neutralizing antibody titres measured by plaque reduction assay versus titres measured with a pseudotype assay 
at both $50 \%$ and $90 \%$ inhibition of virus entry end-points. Correlation coefficients for live virus versus $50 \%$ and $90 \%$ pseudovirus inhibition were 0.78 and 0.69 respectively (Temperton et al. 2005). Zhang et al. studied temporal changes in $\mathrm{N}$ protein-specific (by quantitative ELISA) and S glycoprotein-specific neutralizing antibody responses (by SARS-CoV pseudotype assay) in infected patients who had either recovered from or succumbed to SARS-CoV infection (Zhang et al. 2006). A good correlation between strong antibody responses to $\mathrm{N}$ and $\mathrm{S}$ proteins and disease outcome of infected individuals was shown. In a large comprehensive study on 128 SARS convalescent patient sera, strong T-cell responses were shown to correlate significantly $(p<0.05)$ with higher neutralizing antibody titer as measured by an MLV(SARS) assay (Li et al. 2008).

\subsection{Conclusion}

In conclusion, SARS pseudotypes will enable much work that currently needs to be performed in Category III/IV laboratories to be undertaken with pseudotypes in a Category II environment. They represent a highly flexible and sensitive system for the evaluation of neutralizing antibodies against the spike surface glycoprotein.

Given that Research Councils UK and the Health Protection Agency (HPA) are currently inviting consultation on the UK national need for investment in high containment laboratories, it should be emphasized that the application of pseudotypes to highly pathogenic enveloped viruses such as SARS, H5N1 influenza A, rabies, West Nile and other high containment viruses can help to some extent to alleviate the pressure on expensive containment facilities.

Acknowledgments I would like to thank Edward Wright for invaluable assistance in the preparation of the figures, and Robin Weiss for his encouragement and mentorship.

\section{References}

Bermingham A, Heinen P, Iturriza-Gomara M, Gray J, Appleton H, Zambon MC (2004) Laboratory diagnosis of SARS. Philos Trans R Soc Lond 359:1083-1089

Capecchi B, Fasolo A, Alberini I, Baudner B, Crotta S, Temperton NJ, Montomoli E, del Giudice G, Rappuoli R (2008) Use of pseudotyped particles expressing Influenza A/Vietnam/1194/ 2004 hemagglutinin in neutralization assays. In Katz JM (ed) Options for the control of Influenza VI. International Medical, London, pp 303-305

Dye C, Temperton N, Siddell SG (2007) Type I feline coronavirus spike glycoprotein fails to recognize aminopeptidase $\mathrm{N}$ as a functional receptor on feline cell lines. J Gen Virol 88: $1753-1760$

Giroglou T, Cinatl J Jr, Rabenau H, Drosten C, Schwalbe H, Doerr HW, von Laer D (2004) Retroviral vectors pseudotyped with severe acute respiratory syndrome coronavirus S protein. J Virol 78:9007-9015

Han DP, Kim HG, Kim YB, Poon LL, Cho MW (2004) Development of a safe neutralization assay for SARS-CoV and characterization of S-glycoprotein. Virology 326:140-149 
Hattermann K, Muller MA, Nitsche A, Wendt S, Donoso Mantke O, Niedrig M (2005) Susceptibility of different eukaryotic cell lines to SARS-coronavirus. Arch Virol 150:1023-1031

Hofmann H, Hattermann K, Marzi A, Gramberg T, Geier M, Krumbiegel M, Kuate S, Uberla K, Niedrig M, Pohlmann S (2004) S protein of severe acute respiratory syndrome-associated coronavirus mediates entry into hepatoma cell lines and is targeted by neutralizing antibodies in infected patients. J Virol 78:6134-6142

Hofmann H, Pyrc K, van der Hoek L, Geier M, Berkhout B, Pohlmann S (2005) Human coronavirus NL63 employs the severe acute respiratory syndrome coronavirus receptor for cellular entry. Proc Natl Acad Sci USA 102:7988-7993

Kaye M (2006) SARS-associated coronavirus replication in cell lines. Emerg Infect Dis 12: $128-133$

Li CK, Wu H, Yan H, Ma S, Wang L, Zhang M, Tang X, Temperton NJ, Weiss RA, Brenchley JM, Douek DC, Mongkolsapaya J, Tran BH, Lin CL, Screaton GR, Hou JL, McMichael AJ, Xu XN (2008) T cell responses to whole SARS coronavirus in humans. J Immunol 181:5490-5500

Moore MJ, Dorfman T, Li W, Wong SK, Li Y, Kuhn JH, Coderre J, Vasilieva N, Han Z, Greenough TC, Farzan M, Choe H (2004) Retroviruses pseudotyped with the severe acute respiratory syndrome coronavirus spike protein efficiently infect cells expressing angiotensinconverting enzyme 2. J Virol 78:10628-10635

Nie Y, Wang G, Shi X, Zhang H, Qiu Y, He Z, Wang W, Lian G, Yin X, Du L, Ren L, Wang J, He X, Li T, Deng H, Ding M (2004a) Neutralizing antibodies in patients with severe acute respiratory syndrome-associated coronavirus infection. J Infect Dis 190:1119-1126

Nie Y, Wang P, Shi X, Wang G, Chen J, Zheng A, Wang W, Wang Z, Qu X, Luo M, Tan L, Song X, Yin X, Chen J, Ding M, Deng H (2004b) Highly infectious SARS-CoV pseudotyped virus reveals the cell tropism and its correlation with receptor expression. Biochem Biophys Res Commun 321:994-1000

Simmons G, Reeves JD, Rennekamp AJ, Amberg SM, Piefer AJ, Bates P (2004) Characterization of severe acute respiratory syndrome-associated coronavirus (SARS-CoV) spike glycoproteinmediated viral entry. Proc Natl Acad Sci USA 101:4240-4245

Su CY, Wang SY, Shie JJ, Jeng KS, Temperton NJ, Fang JM, Wong CH, Cheng YS (2008) In vitro evaluation of neuraminidase inhibitors using the neuraminidase-dependent release assay of hemagglutinin-pseudotyped viruses. Antiviral Res 79:199-205

Temperton NJ, Wright E (2009) Retroviral pseudotypes. In: Encyclopedia of Life Sciences (ELS). Wiley, Chichester. DOI: 10.1002/9780470015902.a0021549

Temperton NJ, Chan PK, Simmons G, Zambon MC, Tedder RS, Takeuchi Y, Weiss RA (2005) Longitudinally profiling neutralizing antibody response to SARS coronavirus with pseudotypes. Emerg Infect Dis 11:411-416

Temperton NJ, Hoschler K, Major D, Nicolson C, Manvell R, Hien VM, Ha DQ, de Jong MD, Zambon M, Weiss RA (2007) A sensitive retroviral pseudotype assay for influenza H5N1 neutralizing antibodies. Influenza Other Respir Viruses 1:105-112

Temperton NJ, Capecchi B, Rappuoli R, de Jong MD, Takeuchi Y, Weiss RA (2008) Measurement of neutralizing antibody responses to influenza H5N1 clade 1 A/Viet Nam/1194/04 and clade $2 \mathrm{~A} /$ Indonesia/5/05 hemagglutinin using a sensitive high throughput luciferase-based retroviral pseudotype assay. In Katz JM (ed) Options for the Control of Influenza VI. International Medical Press, London, pp 94-96

Wang SY, Su CY, Temperton NJ, Jeng KS, Wong CH, Cheng YS (2008) HA-pseudotyped retroviral vectors for screening and evaluation of anti-flu inhibitors. In Katz JM (ed) Options for the Control of Influenza VI. International Medical Press, London, pp 472-474

Wright E, Temperton NJ, Marston DA, McElhinney LM, Fooks AR, Weiss RA (2008) Investigating antibody neutralization of lyssaviruses using lentiviral pseudotypes: a cross-species comparison. J Gen Virol 89:2204-2213

Wu HS, Chiu SC, Tseng TC, Lin SF, Lin JH, Hsu YH, Wang MC, Lin TL, Yang WZ, Ferng TL, Huang KH, Hsu LC, Lee LL, Yang JY, Chen HY, Su SP, Yang SY, Lin SY, Lin TH, Su IS 
(2004) Serologic and molecular biologic methods for SARS-associated coronavirus infection, Taiwan. Emerg Infect Dis 10:304-310

Zhang L, Zhang F, Yu W, He T, Yu J, Yi CE, Ba L, Li W, Farzan M, Chen Z, Yuen KY, Ho D (2006) Antibody responses against SARS coronavirus are correlated with disease outcome of infected individuals. J Med Virol 78:1-8

Zhu Z, Chakraborti S, He Y, Roberts A, Sheahan T, Xiao X, Hensley LE, Prabakaran P, Rockx B, Sidorov IA, Corti D, Vogel L, Feng Y, Kim JO, Wang LF, Baric R, Lanzavecchia A, Curtis KM, Nabel GJ, Subbarao K, Jiang S, Dimitrov DS (2007) Potent cross-reactive neutralization of SARS coronavirus isolates by human monoclonal antibodies. Proc Natl Acad Sci USA 104:12123-12128 